\title{
Artificial Intelligence-Based Inferior Vena Cava Images under Dezocine Anesthesia in Detection of Bile Duct Injury after Laparoscopic Cholecystectomy
}

\author{
Yantao Chen $\mathbb{D},{ }^{1}$ Qinyao Zeng $\mathbb{D},{ }^{1}$ Biao Feng $\mathbb{D}$, ${ }^{1}$ and Haixia Xiong $\mathbb{D}^{2}$ \\ ${ }^{1}$ Department of Anesthesiology, Xiangya Changde Hospital, Changde 415000, Hunan, China \\ ${ }^{2}$ Department of Anesthesiology, Affiliated Hospital of Jianghan University, Wuhan 430015, Hubei, China \\ Correspondence should be addressed to Haixia Xiong; khalid@ahu.edu.cn
}

Received 9 July 2021; Revised 14 August 2021; Accepted 16 August 2021; Published 6 September 2021

Academic Editor: Gustavo Ramirez

Copyright (c) 2021 Yantao Chen et al. This is an open access article distributed under the Creative Commons Attribution License, which permits unrestricted use, distribution, and reproduction in any medium, provided the original work is properly cited.

\begin{abstract}
This study focused on the segmentation effects of an artificial intelligence-based algorithm of CT images, to detect the bile duct injury (BDI) after laparoscopic cholecystectomy (LC) under dezocine anesthesia. This study was based on the maximum betweenclass variance (Otsu) algorithm; it introduced the image grayscale mapping method to increase the accuracy of the target area segmentation within the CT image and compare the segmentation effect with the threshold segmentation and the regional growth segmentation algorithm. 46 patients treated with laparoscopic cholecystectomy (LC) were used as research objects, and all patients were inspected in the abdominal CT examination. According to the anesthetic drug selection, patients were divided into control group (conventional anesthesia) and dezocine group (conventional anesthesia + dezocine), with 23 cases in each group. And it compared the difference between the respiratory recovery time, the wake time, the tube time, and the postoperative $3,6,12$, and $24 \mathrm{~h}$ after surgery, and complication after LC evaluation of bile duct injury (BDI). It was found that the algorithm in this study can segment the target area in CT image accurately. Compared with the threshold segmentation and region growing segmentation algorithms, its Dice similarity coefficient (DSC) and Jaccard similarity coefficient (JSC) were higher $(P<0.05)$. There was no statistically significant difference in postoperative spontaneous breathing recovery time, wake-up time, and extubation time between the dezocine group and the control group $(P>0.05)$, but in the dezocine group, the visual analogue scale (VAS) scores at $3,6,12$, and 24 hours after the surgery were lower $(P<0.05) .27$ patients developed BDI after the surgery, and they were classified as per the Strasberg classification standard. It was found that 6 cases were evaluated as type A, 4 cases were type B, 2 cases were type C, 6 cases were type D, and 9 cases were type E. It was concluded that the algorithm in this study can segment the target area of the CT image accurately, assisting the doctor in diagnosis. The use of dezocine before LC can effectively relieve patients' postoperative pain. This study provides a basis for the diagnosis and treatment of gallbladder disease and the detection of complications.
\end{abstract}

\section{Introduction}

Hepatobiliary diseases are common, especially cholelithiasis. With the improvement of living standards, the incidence of cholelithiasis still increases resulting from the changes in eating habits [1]. The incidence increases with age, and females are predominantly affected. Gallstones were mainly pigment stones in the liver and bile ducts, but now they are mainly cholesterol stones, which are also the main components of gallbladder stones [2]. In recent years, laparoscopy technology is constantly developing. It minimizes the hospital stay, reduces trauma, and increases the surgical field of view. Additionally, it allows simultaneous observations from multiple angles and multiple fields of view [3]. Clinically, laparoscopic cholecystectomy (LC) has gradually replaced open cholecystectomy (OC) and open mini-cholecystectomy $(\mathrm{OM})$, becoming the best surgical method for clinical treatment of benign gallbladder diseases [4]. Statistics have shown that the incidence of complications after the LC surgery is approximately $9 \%$ to $17 \%$, and bile duct injury (BDI) is one of the most serious complications. The incidence of BDI after the LC surgery is $0.4 \%$ to $1.3 \%$, higher 
than $0.1 \%$ and $0.2 \%$ after the OC surgery [5]. Minor BDI will prolong the recovery time of the patient. In severe cases, multiple operations are required, which will seriously reduce the quality of life of the patient after surgery and even lead to death [6]. Dezocine is a mixed opioid receptor agonistantagonist used in clinical anesthesia and postoperative analgesia.

Computer tomography $(\mathrm{CT})$ is a rapidly developed imaging technology, and doctors read CT images to diagnose gallbladder diseases and evaluate postoperative complications [7]. However, the gallbladder is located in the gallbladder fossa of the liver and only occupies a small part of the abdominal CT image, and thus it is necessary to segment the gallbladder for easier observation [8]. In the past, the doctor manually segmented the gallbladder boundary in the CT image and finally merged the segmentation results to obtain the three-dimensional results. This method requires strong professional knowledge, and it is time-consuming [9]. Therefore, image segmenting algorithm assists doctors in clinical diagnosis and treatment. Currently threshold segmentation, regional growth, split merge segments, and cluster-based segmentation methods are commonly used for CT image segmentation. Threshold segmentation is a common method, but the CT image of the target and background grayscale gap is poor [10]. Regional growth and split merge segmentation method is simple to separately apply to small structures, which are not ideal for complex picture segmentation, which is easy to generate segmentation [11]. Cluster-based segmentation methods cannot be evaluated between image samples [12]. Maximum betweenclass variance (Otsu) algorithm can convert CT images to a binary image, which is better to divide the CT image, but the target area within the class cannot be distinguished [13], so it is needed for optimization.

In this study, an image segmentation algorithm was used to process CT images. With the segmentation of inferior vena cava (IVC) in abdominal CT as an example, the image segmentation ability of the improved Otsu algorithm was verified. Then, the analgesia effects of dezocine in LC the BDI after the LC surgery were evaluated, expected to provide reference for the treatment of hepatobiliary diseases.

\section{Materials and Methods}

2.1. Research Subjects. In this study, 46 patients admitted to the hospital from June 2019 to September 2020 undergoing LC surgery were selected, including 19 males and 27 females, aged 21-67 years. They were randomly divided into observation group and reference group, with 23 cases in each group. There was no statistically significant difference in the general data such as gender and age between the two groups $(P>0.05)$, and they were comparable. This study has been approved by the ethic committee of the hospital. All patients and their families understood the study and had signed an informed consent form.

The subjects were selected as the following inclusion criteria: (I) patients without mental disorder; (II) patients with normal organ function; (III) patients without concurrent malignant disease; and (IV) patients who had not taken opioid analgesics.

Exclusion criteria were as follows: (I) patients with a history of mental illness; (II) patients with severe cardiovascular and cerebrovascular diseases; (III) patients who had taken opioid analgesics for a long time; and (IV) patients with liver and kidney dysfunction.

At the same time, the general data and postoperative follow-up data of 27 patients admitted to the Affiliated Hospital of Jianghan University from September 2015 to September 2020, who had BDI after undergoing LC surgery, were collected, to evaluate the role of CT images in diagnosing BDI after the LC surgery.

2.2. CT Examination. In this study, a 16-slice spiral CT scanner from Siemens, Germany, is used to scan the patient's upper abdomen. For double-slice spiral CT tomography, the scanning parameters are set as follows: the tube voltage is $180 \mathrm{kV}$, tube current is $45 \mathrm{mAs}$, slice thickness is $5.0 \mathrm{~mm}$, and screw pitch is $1.5 \mathrm{~mm}$. 2 experienced physicians independently read the CT images in a blind manner.

2.3. Segmentation Algorithm of CT Images. The Otsu algorithm is widely used because of its fast speed, good segmentation effects, and strong scalability.

Because the gray value of the image collected by the device has a large difference, it is mapped to $[0,255]$ first.

$$
Y(a, b)=\frac{X(a, b)-\min (X)}{\max (X)-\min (X)} \times 255,
$$

where $X$ represents the original image, $Y$ represents the output image, $(a, b)$ is the coordinate on the $\operatorname{map}, \min (X)$ is the minimum gray value of the original image, and $\max (X)$ is the maximum gray value.

Suppose the image has $N$ gray levels, $m_{i}$ is the number of pixels contained in the $i \in[0, N-1]$ gray level, and $M$ is the total number of pixels. Then, the equation below is obtained:

$$
M=m_{0}+m_{2}+\cdots+m_{(N-1)}=\sum_{i=0}^{N-1} m_{i} .
$$

$P_{i}$ is the probability of the $i$-th gray level, expressed as $P_{i}=m_{i} / M$, and then, there is $\sum_{i=0}^{N-1} P=1$. A threshold $t$ is set, and the image is divided into two classes of $F_{0}$ and $F_{1}$ according to the gray level, where $F_{0}=\{0,2,3, \ldots, x\}$. $p_{0}$ and $p_{1}$ represent the probability of $F_{0}$ and $F_{1}$, respectively.

$$
\begin{aligned}
& p_{0}=P_{r}\left(F_{0}\right)=\sum_{i=0}^{t} P_{i}=p(t), \\
& p_{1}=P_{r}\left(F_{1}\right)=\sum_{i=t+1}^{N-1} P_{i}=1-p(t) .
\end{aligned}
$$

The mean gray values of $F_{0}$ and $F_{1}$ classes are expressed as follows: 


$$
\begin{aligned}
& u_{0}=\sum_{i=0}^{t} i \times P_{r}\left(i \mid F_{0}\right)=\sum_{i=0}^{t} \frac{j P_{i}}{p_{0}}=\frac{u(t)}{p_{0}}, \\
& u_{1}=\sum_{i=t+1}^{N-1} i \times P_{r}\left(i \mid F_{1}\right)=\sum_{i=t+1}^{N-1} \frac{j P_{i}}{p_{1}}=\frac{u_{T}-u(t)}{1-p(t)},
\end{aligned}
$$

where

$$
\begin{aligned}
u_{T} & =u(N-1)=\sum_{i=1}^{N-1} i P_{i}, \\
p(t) & =\sum_{i=0}^{t} P_{i}, \\
u(t) & =\sum_{i=0}^{t} i P_{i}, \\
p_{0}+p_{1} & =1, \\
p_{0} u_{0}+p_{1} u_{1} & =u_{T} .
\end{aligned}
$$
follows:

$$
\begin{aligned}
& \sigma_{0}^{2}=\sum_{i=o}^{t}\left(i-u_{0}\right)^{2} P_{r}\left(i \mid F_{0}\right)=\sum_{i=0}^{t}\left(i-u_{0}\right)^{2} \frac{P_{i}}{p_{0}} \\
& \sigma_{1}^{2}=\sum_{i=t+1}^{N-1}\left(i-u_{1}\right)^{2} P_{r}\left(i \mid F_{1}\right)=\sum_{i=t+1}^{N-1}\left(i-u_{1}\right)^{2} \frac{P_{i}}{p_{1}}
\end{aligned}
$$

In order to find the optimal threshold, three evaluation functions are set:

$$
\begin{aligned}
& x=\frac{\sigma_{B}^{2}}{\sigma_{W}^{2}}, \\
& y=\frac{\sigma_{T}^{2}}{\sigma_{W}^{2}}, \\
& z=\frac{\sigma_{B}^{2}}{\sigma_{T}^{2}},
\end{aligned}
$$

where $\sigma_{B}^{2}, \sigma_{W}^{2}$, and $\sigma_{T}^{2}$ represent the variance between the classes, the variance within the classes, and the total variance, expressed as follows:

$$
\begin{aligned}
\sigma_{B}^{2} & =p_{0}\left(u_{0}-u_{T}\right)^{2}+p_{0}\left(u_{1}-u_{T}\right)^{2} \\
& =p_{0} u_{0}^{2}+p_{1} u_{1}^{2}-u_{T}^{2} \\
\sigma_{W}^{2} & =p_{0} \sigma_{0}^{2}+p_{1} \sigma_{1}^{2} \\
\sigma_{T}^{2} & =\sum_{i=0}^{N-1}\left(i-u_{T}\right)^{2} P_{i}
\end{aligned}
$$

At this time, the problem is converted into how to find an optimal $t$, so that the three discriminant functions are the largest. Because $\sigma_{T}^{2}=\sigma_{W}^{2}+\sigma_{B}^{2}$, the relationship between $x, y$, and $z$ can be converted to

$$
\begin{aligned}
& y=x+1, \\
& z=\frac{x}{x+1}, \\
& y=\frac{1}{1-z} .
\end{aligned}
$$

It is noted that the three discriminant functions have the same monotonicity. Because $\sigma_{T}^{2}$ can be calculated quickly, and are independent of $t$, it is the simplest to use $\sigma_{T}^{2}$ as the analysis object. Also, $z=\sigma_{B}^{2} / \sigma_{T}^{2}$, the analysis function is further simplified; that is, $\sigma_{B}^{2}$ is used as the classification discriminant function.

$$
\sigma_{B}^{2}(t)=\frac{\left[u_{T} p(t)-u(t)\right]^{2}}{p(t)[1-p(t)]},
$$

where $u(t)=\sum_{i-0}^{t} j P_{i}, p(t)=\sum_{i=0}^{t} P_{i}, u_{T}=\sum_{i=0}^{N-1} i P_{i}$.

Suppose there is a formula that is proportional to the mean distance difference between various classes and inversely proportional to the distance sum between various classes. When the maximum is taken, $t$ at this time is the optimal threshold.

$$
X(t)=p_{0} p_{1} \frac{\left[P_{0}(t)-P_{B}(t)\right]^{2}}{\delta_{0}(t)+\delta_{1}(t)},
$$

where $P_{0}(t)$ and $P_{B}(t)$ are the targets and $\delta_{0}(t)$ and $\delta_{1}(t)$ are the target and background average variance, respectively.

$$
\begin{aligned}
& P_{0}(t)=\sum_{i=0}^{t} \frac{i P(i)}{p_{0}}, \\
& P_{B}(t)=\sum_{i=t+1}^{N-1} \frac{i P(i)}{p_{1}}, \\
& \delta_{0}(t)=\frac{\sum_{i=0}^{t}\left[i-P_{0}(t)\right]^{2} P(i)}{p_{0}}, \\
& \delta_{1}(t)=\frac{\sum_{i=t+1}^{N-1}\left[i-P_{B}(t)\right]^{2} P(i)}{p_{1}} .
\end{aligned}
$$

The optimal threshold $t$ is the $t$ value when $X(t)$ takes the maximum value, and the optimal threshold $t$ satisfies the following equation:

$$
\sigma_{B}^{2}(t)=\max \left\{\sigma_{B}^{2}(t)\right\} .
$$

2.4. Anesthesia Methods. The patients were randomly divided into dezocine group and control group according to the order of visits. Before the surgery, $0.1 \mathrm{~g}$ phenobarbital and $0.5 \mathrm{~g}$ atropine $0.5 \mathrm{~g}$ were intramuscularly injected. After the venous access was opened, the blood pressure, heart rate, and pulse oxygen saturation were routinely monitored. In both groups, patients were injected with $0.003 \mathrm{mg} / \mathrm{kg}$ fentanyl, $0.08 \mathrm{mg} / \mathrm{kg}$ atracurium, and $2 \mathrm{mg} / \mathrm{kg}$ propofol for induction intubation. During the operation, isoflurane was 
continuously given and Kasining was administered depending on the circumstances. In the dezocine group, $0.1 \mathrm{mg} / \mathrm{kg}$ dezocine was given during the induction. After the patient's breathing was restored, the tracheal tube was removed if the extubation indications were met, and then they were sent back to the ward after being fully awake.

2.5. Evaluation of the Segmentation Effects. In order to evaluate the segmentation effects of the algorithm in this study, threshold segmentation and region growing segmentation algorithms are introduced to compare with the algorithm in this study. The segmentation effects were evaluated factoring into the Dice similarity coefficient (DSC) and Jaccard similarity coefficient (JSC).

$$
\begin{aligned}
\text { DSC } & =\frac{2|A \cap B|}{|A|+|B|} \times 100 \%, \\
\text { JSC } & =\frac{|A \cap B|}{|A \cup B|} \times 100 \%,
\end{aligned}
$$

where $A$ is the manual segmentation results and $B$ is the segmentation results by the algorithm in the study. $A \cap B$ is the intersection of $A$ and $B$, and $A \cup B$ is the union of $A$ and $B$. If the DSC and JSC indicators are closer to 1 , it means that the two images are more similar, indicating better segmentation effects.

\subsection{Observation Index of Anesthesia Effects of Dezocine.} Relevant indexes were recorded, including spontaneous breathing recovery time, wake-up time, and extubation time, and the analgesic effects were evaluated at 3, 6, 12, and 24 hours after surgery. The visual analogue (VAS) score was used to evaluate the analgesic effects: 0 meant no pain, and 10 meant pain was intolerable.

2.7. Evaluation of BDI. Usually, BDI occurs 1 to 2 weeks after the surgery. The patient has one or more symptoms of abdominal pain, peritonitis, cholangitis, and sudden high fever. In this study, as per the Strasberg classification, BDI was classified into types A to E: type A: there was bile leakage from the small bile duct, but the continuity of the extrahepatic bile duct was not destroyed, such as the vagal bile duct leak in the gallbladder bed; type B: the obstruction of the branches of the biliary tree, and the more common was the accidental clipping of the right accessory hepatic duct; type C: the open bile leakage caused by the cut injury to the right accessory hepatic duct; type D: the bile leakage caused by the side wall injury of the extrahepatic bile duct; and type E: injury related to the common bile duct.

2.8. Statistical Methods. SPSS 20.0 was used to process the data. The measurement data were expressed as mean\pm standard deviation $(\bar{x} \pm s)$, and $t$-test was used. The count data adopted chi-square test. $P<0.05$ was the threshold for significance.

\section{Results}

3.1. Image Processing Results. All patients accepted CT examinations, and the collected CT images were standardized and normalized. The improved Otsu algorithm was used to segment the IVC, and the expert manual segmentation results were used as the standard. Figure 1 shows the CT segmentation results of two patients. The target area and expert manual segmentation of the Otsu algorithm were most closely related, and the division of the threshold segmentation method was the largest. It showed that the improved Otsu algorithm used in this study could be divided more accurately.

3.2. Image Segmentation Results. After the original image was segmented using the algorithm, the DSC values were compared with those of the manually segmented image by the expert, DSC values of different algorithms are shown in Figure 2, the DSC values of improved Otsu, threshold, and region growing were $(0.85 \pm 0.092),(0.59 \pm 0.042)$, and $(0.63 \pm 0.071)$, the DSC values of the improved Otsu were higher than the threshold segmentation and region growth segmentation, and the difference was statistically significant $(P<0.05)$.

After the original image was segmented using the algorithm, the JSC values were compared with those of the manually segmented image by the expert; JSC values of different algorithms are shown in Figure 3. The JSC values of improved Otsu, threshold, and region growing were $(0.81 \pm 0.089),(0.55 \pm 0.048)$, and $(0.58 \pm 0.053)$, the JSC values of the improved Otsu were higher than the threshold segmentation and region growth segmentation, and the difference was statistically significant $(P<0.05)$.

3.3. Anesthesia Effects of Dezocine. Figure 4 shows the postoperative spontaneous breathing recovery time; Figure 5 shows the wake-up time; Figure 6 shows the extubation time; and Figure 7 shows the postoperative analgesic effects. There was no significant difference in spontaneous breathing recovery time, wake-up time, and extubation time between the dezocine group and the control group, $P>0.05$, indicating that the use of dezocine before the LC surgery had no effects on the patient's recovery.

The VAS scores of the two groups were compared, and the VAS scores at $3 \mathrm{~h}, 6 \mathrm{~h}, 12 \mathrm{~h}$, and $24 \mathrm{~h}$ after surgery in the control group were $(3.27 \pm 0.29),(5.12 \pm 0.51),(5.48 \pm 0.51)$, and $(4.92 \pm 0.47)$, and the VAS scores at $3 \mathrm{~h}, 6 \mathrm{~h}, 12 \mathrm{~h}$, and $24 \mathrm{~h}$ after surgery in the dezocine group were $(1.23 \pm 0.18)$, $(2.30 \pm 0.25),(2.62 \pm 0.19)$, and $(1.81 \pm 0.16)$; it can be concluded that the postoperative $3 \mathrm{~h}, 6 \mathrm{~h}, 12 \mathrm{~h}$, and $24 \mathrm{~h}$ VAS scores were lower than the control group $(P<0.05)$.

3.4. Evaluation of BDI after the LC Surgery. After the surgery, 27 patients developed BDI. They were classified as per the Strasberg classification standard. Of them, 6 cases were evaluated as type A, 4 cases were type B, 2 cases were type C, 6 cases were type D, and 9 cases were type E (Figure 8). 

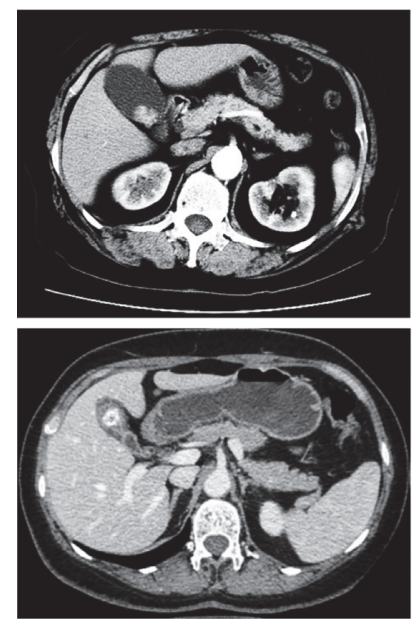

Original image
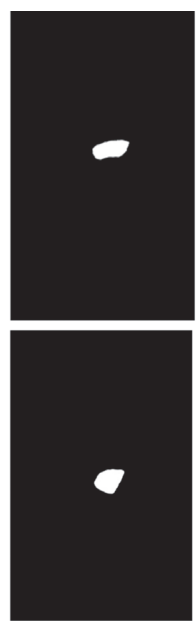

Manual segmentation
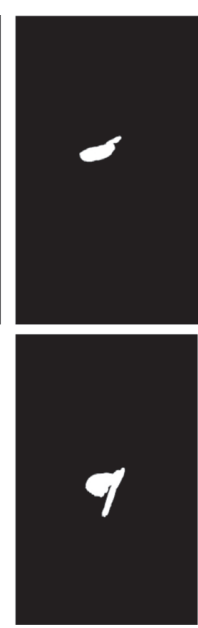

Threshold
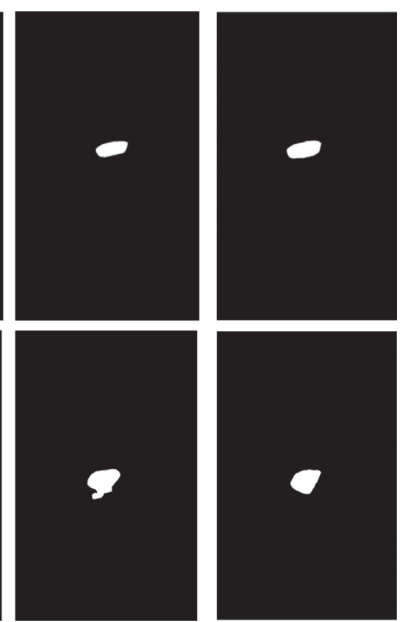

Region growing

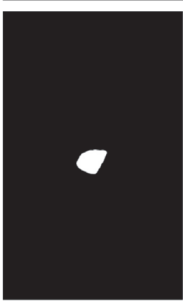

Improved OTSU

FIGURE 1: Segmentation results of different algorithms.

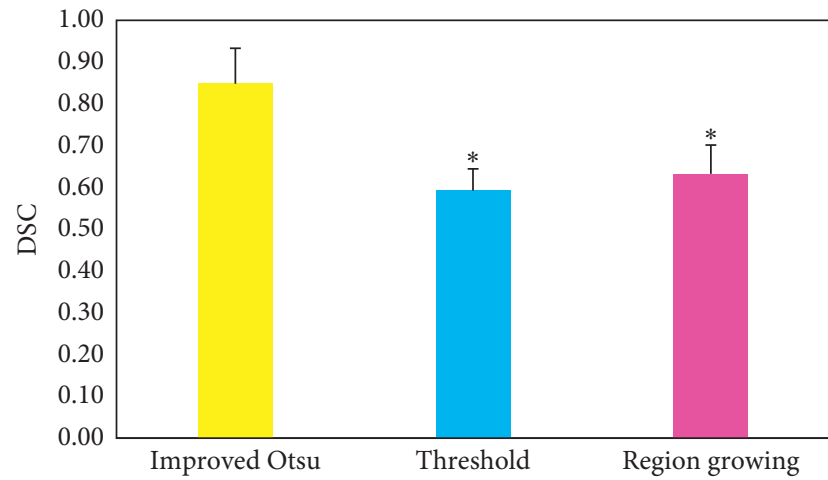

FIgURE 2: Comparison of DSC values of the three algorithms $\left({ }^{*}\right.$ indicates that there was a significant difference compared to improved Otsu, $P<0.05)$.

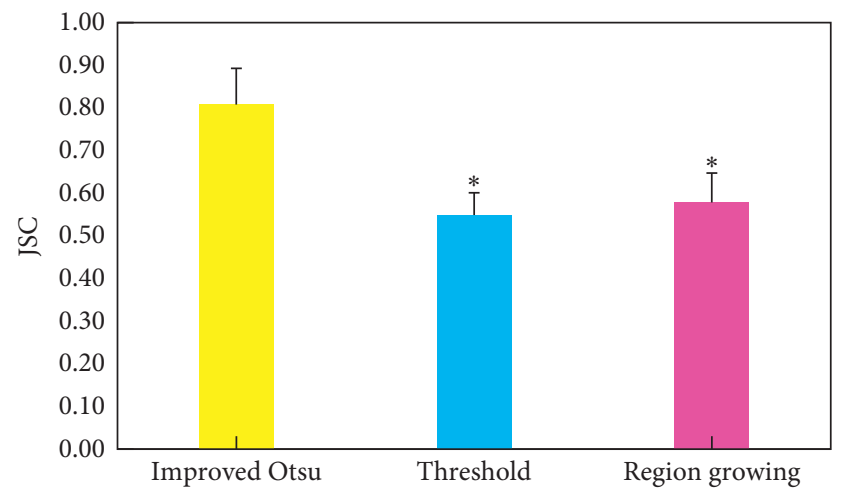

FIgURE 3: Comparison of JSC values of the three algorithms ( ${ }^{*}$ indicates that there was a significant difference compared to improved Otsu, $P<0.05)$.

\section{Discussion}

Compared with OC, LC has become the first choice for gallstones and benign gallbladder diseases due to less trauma, less pain, faster healing, and shorter hospital stay

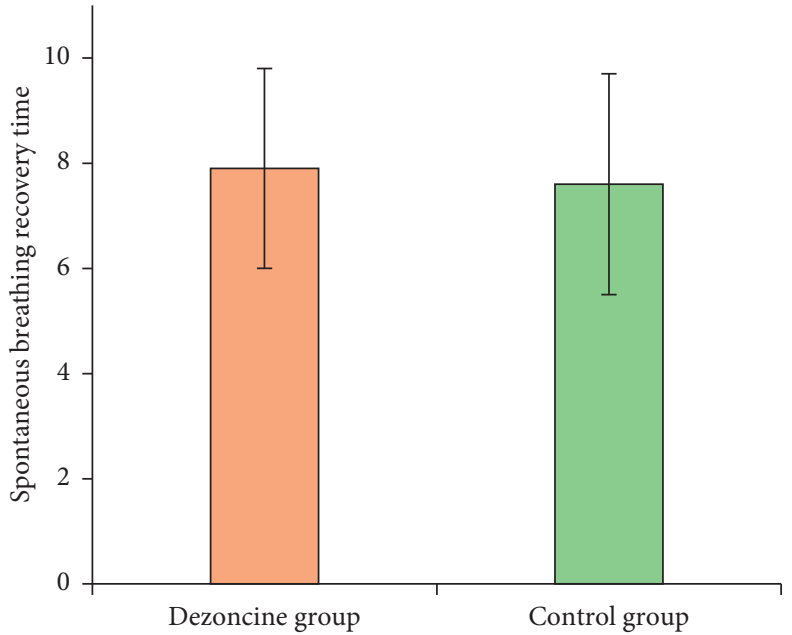

Figure 4: Comparison of the recovery time of spontaneous breathing between the two groups.

[14]. However, the postoperative complications reduce the quality of life, especially the BDI. In some areas where LC is not yet mature, the incidence of BDI is higher [15]. BDI can cause bile duct stenosis, bile duct obstruction, bile duct infection, and long-term complications, such as liver cirrhosis and liver atrophy [16]. The causes of BDI are complicated, including surgical factors, such as conventional cholecystectomy, mainly LC, and other interventions, and nonsurgical factors, such as interventional or endoscopic treatment [17-19]. During the LC surgery, BDI may be caused by LC which may be caused by subjective factors, such as the lack of operating skills and experience of the doctor, the insufficient standardization of the operation, the failure of the surgeon to accurately assess the difficulty of the operation, and the insufficient skills of the assistant in mirror support. It can also arise from objective reasons. For example, laparoscopy technology itself has certain limitations; when operations are performed on the screen, it is 




Figure 5: Comparison of wake-up time between the two groups of patients.

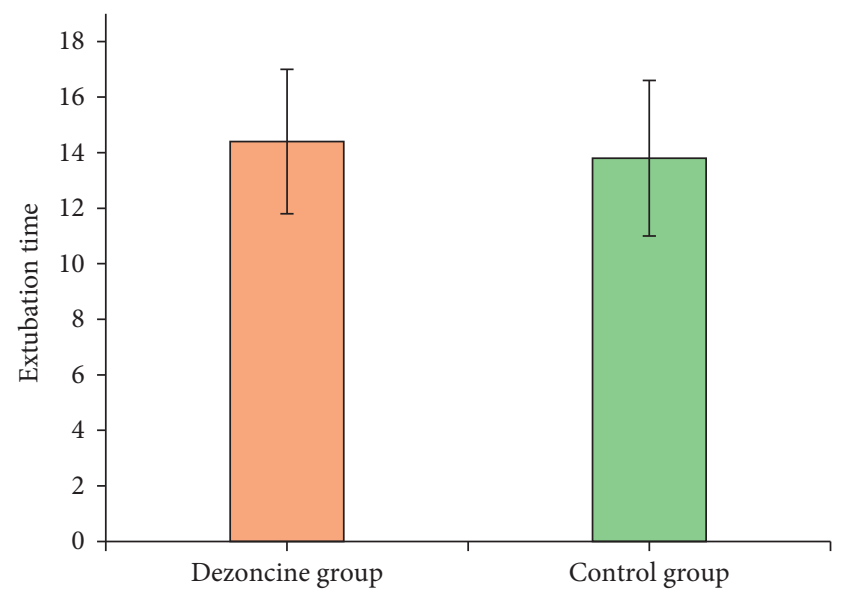

FIGURE 6: Comparison of extubation time between the two groups of patients.

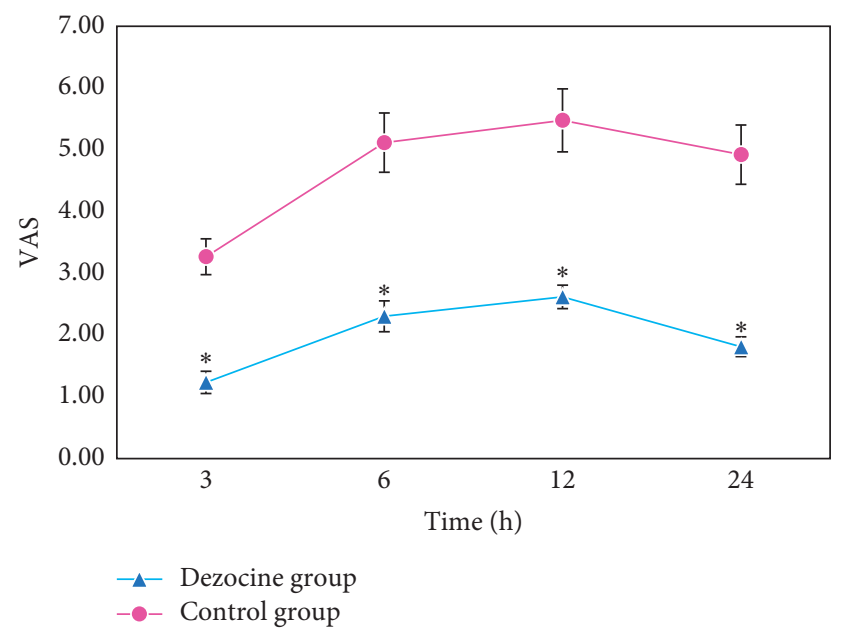

FIGURE 7: Comparison of VAS scores between the two groups of patients $\left({ }^{*}\right.$ indicates that there was a significant difference compared to the control group, $P<0.05)$.

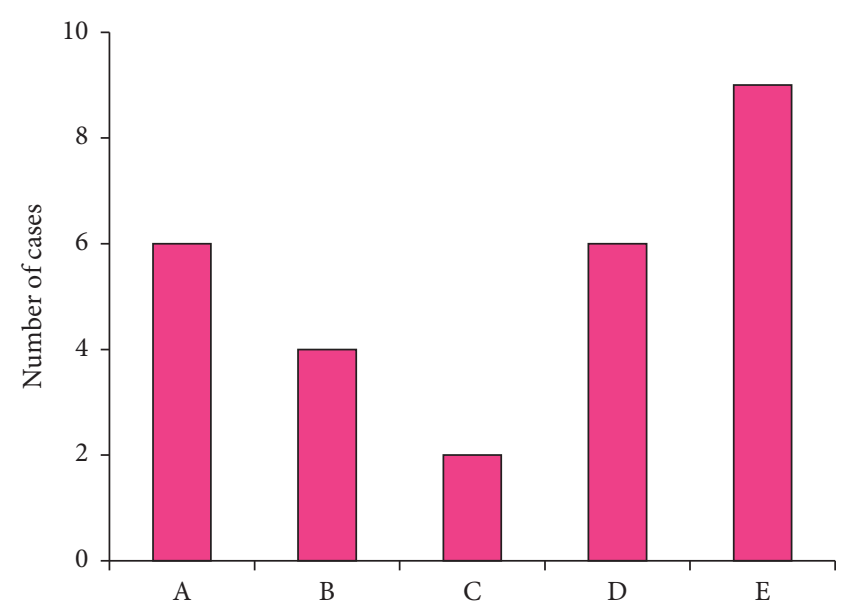

FIGURE 8: Strasberg classification results of patients with BDI after the LC surgery.

sometimes difficult to determine the accurate anatomical position, because the surgeon cannot directly touch the operation area; and obese patients will also affect the surgical field of vision to a certain extent [20-22]. In addition, changes in the shape and position of the gallbladder will increase the risk of BDI [23].

LC requires general anesthesia, and patients will suffer from pain after the LC surgery. Post-LC pain mainly occurs 24 hours after surgery, manifesting as incision pain, visceral pain, and involved shoulder pain [24]. Pain may be caused by excessive traction on the patient's peritoneum during the operation, or incomplete gas release of the pneumoperitoneum after the operation, which seriously affects the recovery of the patient after the operation and increases the occurrence of postoperative complications [25]. Dezocine is an analgesic that has agonistic effects on $K$ receptor $u$. $u$ receptor plays an important role in sedation and alleviating labor pain and mild respiratory depression. It is often used for postoperative analgesia and cancer pain. However, adverse reactions will occur at a large dose. Dezocine is an opioid receptor agonist-antagonist. On the one hand, the drug can be used in spinal analgesia, and on the other hand, it is safe to use. Studies have pointed out that, when the drug is used alone for postoperative analgesia, it requires a large dosage, which may cause adverse reactions, such as drowsiness and dizziness after the operation. It is recommended to use it in combination with other analgesics to reduce the incidence of adverse reactions [26, 27].

Artificial intelligence algorithms have been widely used in the medical field. For example, Guo et al. used ELM and other technologies to identify errors in the uneven distribution report and achieved excellent results [28]. In this study, the Otsu algorithm was optimized, and the improved Otsu algorithm was found to be accurate in segmenting the target area of CT images with the manual segmentation results by the expert as the standard. Compared with the threshold segmentation and region growing segmentation algorithms, its DSC and JSC values were higher, indicating that the improved algorithm had good segmentation effects, which can be used in clinics for the diagnosis and treatment 
of gallbladder diseases and the detection of BDI. After the LC surgery, 27 patients developed BDI. They were classified as per the Strasberg classification standard. It was found that 6 cases were evaluated as type A, 4 cases were type B, 2 cases were type C, 6 cases were type $\mathrm{D}$, and 9 cases were type E. There was no significant difference in the postoperative spontaneous breathing recovery time, wake-up time, and extubation time between the dezocine group and the control group $(P>0.05)$, which proved that the use of dezocine did not have an impact on the patient's recovery. It is believed that the effects of analgesia treatment after the pain occurs are often not good. Preventive analgesia is to take measures in advance to prevent the pain being transmitted to the central nervous system, thereby eliminating or reducing postoperative pain. It not only has obvious analgesia effects, but also requires a low dosage. What is more, the incidence of complications is low. In this study, the VAS scores of the dezocine group at $3,6,12$, and 24 hours after surgery were lower than those of the control group, proving that dezocine was safe and effective for preoperative anesthesia to relieve pain in patients.

\section{Conclusion}

In this study, the Otsu algorithm was optimized and the improved algorithm was used to segment the target area in $\mathrm{CT}$ images. It was found to be accurate in segmenting CT images and the results were close to the manual segmentation results, proving that it can assist doctors in the diagnosis and treatment of gallbladder diseases. Moreover, the use of dezocine anesthesia before LC can alleviate the postoperative pain of patients. However, some limitations in the study should be noted. The subjects are form 1 hospital, and the sample size is small, which may reduce the power of the study. In the follow-up, an expanded sample size is required to strengthen the findings of the study. In conclusion, this study provides a basis for the diagnosis and treatment of gallbladder disease and the detection of complications.

\section{Data Availability}

The data used to support the findings of this study are available from the corresponding author upon request.

\section{Conflicts of Interest}

The authors declare no conflicts of interest.

\section{References}

[1] A. D. Ciaula, G. Garruti, G. Frühbeck et al., "The role of diet in the pathogenesis of cholesterol gallstones," Current Medicinal Chemistry, vol. 26, no. 19, pp. 3620-3638, 2019.

[2] M. Ibrahim, S. Sarvepalli, G. Morris-Stiff et al., "Gallstones: watch and wait, or intervene?" Cleveland Clinic Journal of Medicine, vol. 85, no. 4, pp. 323-331, 2018.

[3] D. Wang, T. Dong, Y. Shao, T. Gu, Y. Xu, and Y. Jiang, "Laparoscopy versus open appendectomy for elderly patients, a meta-analysis and systematic review," BMC Surgery, vol. 19, no. 1, 2019.

[4] V. Gupta and G. Jain, "Safe laparoscopic cholecystectomy: adoption of universal culture of safety in cholecystectomy," World Journal of Gastrointestinal Surgery, vol. 11, no. 2, pp. 62-84, 2019.

[5] F. W. V. D. Graaf, I. Zaïmi, L. P. S. Stassen, and J. F. Lange, "Safe laparoscopic cholecystectomy: a systematic review of bile duct injury prevention," International Journal of Surgery, vol. 60, pp. 164-172, 2018.

[6] M. Visentin, D. Lenggenhager, Z. Gai, and G. A. U. Kullak, "Drug-induced bile duct injury," Biochimica et Biophysica Acta - Molecular Basis of Disease, vol. 1864, no. 4, pp. 14981506, 2018.

[7] P. Grewal and J. Ahmad, "Bile duct injury due to drug-induced liver injury," Current Hepatology Reports, vol. 18, no. 3, pp. 269-273, 2019.

[8] J. Görich and S. Buß, "Computed tomography angiography," Deutsches Arzteblatt international, vol. 116, no. 26, 2019.

[9] F. P. An and Z. W. Liu, "Medical image segmentation algorithm based on feedback mechanism CNN," Contrast Media and Molecular Imaging, vol. 2019, Article ID 6134942, 13 pages, 2019.

[10] L. Friedli, D. Kloukos, G. Kanavakis, D. Halazonetis, and N. Gkantidis, "The effect of threshold level on bone segmentation of cranial base structures from CT and CBCT images," Scientific Reports, vol. 10, no. 1, Article ID 7361, 2020.

[11] H. H. Duan, J. Gong, X. W. Sun, and S. D. Nie, "Region growing algorithm combined with morphology and skeleton analysis for segmenting airway tree in CT images," Journal of X-Ray Science and Technology, vol. 28, no. 2, pp. 311-331, 2020.

[12] G. Delso, K. Zeimpekis, M. Carl, F. Wiesinger, M. Hüllner, and P. Veit-Haibach, "Cluster-based segmentation of dualecho ultra-short echo time images for PET/MR bone localization," EJNMMI Physics, vol. 1, no. 1, 2014.

[13] W. K. P. Barros, L. A. Dias, and M. A. C. Fernandes, "Fully parallel implementation of Otsu automatic image thresholding algorithm on FPGA," Sensors, vol. 21, no. 12, Article ID 4151, 2021.

[14] B. Khorram and M. Yazdi, "A new optimized thresholding method using ant colony algorithm for MR brain image segmentation," Journal of Digital Imaging, vol. 32, no. 1, pp. 162-174, 2019.

[15] B. W. Renz, F. Bösch, and M. K. Angele, "Bile duct injury after cholecystectomy: surgical therapy," Visceral Medicine, vol. 33, no. 3, pp. 184-190, 2017.

[16] A. M. Schreuder, O. R. Busch, M. G. Besselink et al., "Longterm impact of iatrogenic bile duct injury," Digestive Surgery, vol. 37, no. 1, pp. 10-21, 2020.

[17] L. B. Michael, D. J. Deziel, D. A. Telem et al., "Safe cholecystectomy multi-society practice guideline and state-of-theart consensus conference on prevention of bile duct injury during cholecystectomy," Surgical Endoscopy, vol. 34, no. 7, pp. 2827-2855, 2020.

[18] S. Sharma, A. Behari, R. Shukla, M. Dasari, and V. K. Kapoor, "Bile duct injury during laparoscopic cholecystectomy: an Indian e-survey," Annals of Hepato-Biliary-Pancreatic Surgery, vol. 24, no. 4, pp. 469-476, 2020.

[19] B. Xie, H. Luo, X. Yang et al., "Repair of bile duct injury with autologous vein graft and stent," Experimental and Clinical Transplantation: Official Journal of the Middle East Society for Organ Transplantation, vol. 16, no. 6, pp. 696-700, 2018. 
[20] P. M. Terho, A. K. Leppäniemi, and P. J. Mentula, "Laparoscopic cholecystectomy for acute calculous cholecystitis: a retrospective study assessing risk factors for conversion and complications," World Journal of Emergency Surgery, vol. 11, no. $1,2016$.

[21] F. P. Zhong, K. Wang, X. Q. Tan, J. Nie, W. F. Huang, and X. F. Wang, "The optimal timing of laparoscopic cholecystectomy in patients with mild gallstone pancreatitis," Medicine (Baltimore), vol. 98, no. 40, 2019.

[22] E. Ito, A. Takai, Y Imai et al., "Quality of life after singleincision laparoscopic cholecystectomy: a randomized, clinical trial," Surgery, vol. 165, no. 2, pp. 353-359, 2019.

[23] A. Sharma, K. L. Sharma, A. Gupta, A. Yadav, and A. Kumar, "Gallbladder cancer epidemiology, pathogenesis and molecular genetics: recent update," World Journal of Gastroenterology, vol. 23, no. 22, pp. 3978-3998, 2017.

[24] A. W. H. Barazanchi, W. S. MacFater, J. L. Rahiri, S. Tutone, A. G. Hill, and G. P. Joshi, "Evidence-based management of pain after laparoscopic cholecystectomy: a PROSPECT review update," British Journal of Anaesthesia, vol. 121, no. 4, pp. 787-803, 2018.

[25] S. Li, J. Guo, F. Li, Z. Yang, S. Wang, and C. Qin, "Pregabalin can decrease acute pain and morphine consumption in laparoscopic cholecystectomy patients," Medicine (Baltimore), vol. 96, no. 21, 2017.

[26] H. Zhu, Y. Chen, S. Huang, and X. Sun, "Interaction of analgesic effects of dezocine and sufentanil for relief of postoperative pain: a pilot study," Drug Design, Development and Therapy, vol. 14, pp. 4717-4724, 2020.

[27] L. Zhou, Y. Zhang, H. Sun, R. Hu, J. Wang, and G. Xu, "Effect of preemptive dezocine before general anesthesia on postoperative analgesia in patients undergoing laparoscopic cholecystectomy," Medicine (Baltimore), vol. 97, no. 39, 2018.

[28] S. Guo, R. Chen, H. Li, T. Zhang, and Y. Liu, "Identify severity bug report with distribution imbalance by CR-SMOTE and ELM," International Journal of Software Engineering and Knowledge Engineering, vol. 29, no. 2, pp. 139-175, 2019. 